\title{
Erratum to: Use of bisphosphonate and risk of atrial fibrillation in older women with osteoporosis
}

\author{
C. W. Rhee $\cdot$ J. Lee $\cdot$ S. Oh $・$ N. K. Choi $・$ B. J. Park
}

Published online: 15 November 2011

(C) International Osteoporosis Foundation and National Osteoporosis Foundation 2011

\section{Erratum to: Osteoporos Int}

DOI 10.1007/s00198-011-1608-z

In the subsection "Cohort construction" under Methods, the first four sentences of the second paragraph should have read as follows:

Since more than $95 \%$ of the osteoporosis patients revisited their physician for their osteoporosis drug prescriptions within 120 days during the study period, we excluded those who filled their prescription for any osteoporosis medication or had been assigned diagnosis codes for osteoporosis during the period January 1, 2005 to April 30, 2005. By doing so, we constructed a retrospective cohort with newly diagnosed osteoporosis patients who had not taken any medications for osteoporosis. Patients who switched between bisphosphonate and any other medications for osteoporosis were excluded from the study. Additionally, individuals who were diagnosed with cancer (ICD-10 code: C-D), chronic renal failure (ICD10 code: N18), or atrial fibrillation (ICD-10 code: I48) prior to taking osteoporotic drugs were also excluded.

The online version of the original article can be found at http:// dx.doi.org/10.1007/s00198-011-1608-z.

C. W. Rhee $\cdot$ N. K. Choi $\cdot$ B. J. Park $(\bowtie)$

Department of Preventive Medicine, Seoul National University

College of Medicine,

103 Daehangno, Jongno-Gu,

Seoul 110-799, South Korea

e-mail: bjpark@snu.ac.kr

J. Lee · B. J. Park

Medical Research Collaborating Center, Seoul National

University Hospital,

Seoul, South Korea

S. Oh

Department of Internal Medicine, Seoul National University

College of Medicine,

Seoul, South Korea

N. K. Choi

Medical Research Center, Seoul National University,

Seoul, South Korea 